\title{
On the Relationships Between Short-Term Learning and Fluid and Crystallized Intelligence
}

\author{
P. S. Hundal \\ Guru Nanak University \\ John L. Horn \\ University of Denver
}

\begin{abstract}
Eleven indicants of intelligence and 10 measures of short-term learning were studied in a sample of 265 fourteen-year-olds using the inter-battery methods developed by Tucker. The results indicated two broad factors of intelligence, interpreted as fluid intelligence (Gf) and crystallized intelligence (Gc), coordinate with two broad factors of shortterm learning, interpreted as indicating primary memory (PM) and secondary acquisition (SAC). To a considerable extent the learning variables were independent of the indicants of intelligence, thus suggesting (in conformance with previous findings) that intelligence should not be equated with learning over short periods of time. The major variance in common between short-term learning and intelligence variables is linked to meaningful associations and learning mediated by such associations, but to a lesser extent both Gf and Gc involve the span of apprehension of primary memory. The results suggest that acquisition mediated by meaningful associations is more nearly characteristic of $\mathrm{Gc}$ than of Gf, but this may mainly reflect the selection of variables used in this study.
\end{abstract}

\section{Theoretical Introduction}

The ability to learn is often regarded as the sine qua non of intelligence. Perhaps just as often this viewpoint is criticized as oversimplified or precisely wrong, the assertion being that there is no notable relationship between intelli-

APPLIED PSYCHOLOGICAL MEASUREMENT

Vol. 1, No. 1 Winter 1977 pp. 11-21

(c) Copyright 1977 West Publishing Co. gence and ability to learn. (For example, compare the contributions of Buckingham and Colwin with those of Terman and Haggerty in the Pintner, 1945 symposium, or see Guilford, 1967, for review.) An extreme form of the latter view is clearly invalidated by the evidence. But the evidence also clearly indicates that at least some forms of learning bear only a very low relationship to at least some of the well-accepted indicants of intelligence. Thus, if learning is indeed the sine qua non of intelligence, then the reference must be to a particular form of learning, and intelligence also must be defined more narrowly than is common.

At least since the time of Woodrow (1938) it has been known that there are reliably independent dimensions of individual differences in learning performances. The evidence that there are reliably distinct components of conglomerate measures of intelligence dates from an even earlier period, as in the pioneering work of Spearman, Burt and Thurstone. For a variety of learning tasks the general finding has been, also, that when learning is defined as rate of change over relatively short periods of time (one of the longest periods being the 39 days of Woodrow's studies) it has only low (.3 or below) correlations with either separate components of intelligence or a conglomerate measure of the general factor 
(see Birren and Woodruff, 1973; Carroll, 1975; Fleishman and Rich, 1963; Duncanson, 1964; Guilford, 1967; Tucker, 1963 for some results and reviews which provide different perspectives on this theme). The unreliabilities of difference scores and other problems with the learning measures (lack of ceiling in the tasks, for example) often plague efforts to properly interpret these findings. But it seems that when these problems are taken firmly into account, the correlations between well-accepted indicants of intelligence and measures of rate-of-change in learning remain low (e.g., Allison, 1960; Carver and Dubois, 1967; Duncanson, 1964; Harootunion, 1960; Jensen, 1964; Stevenson, Friedrichs and Simpson, 1970; Woodrow, 1938). In particular, the correlations are low relative to those indicating relationships between components of intelligence and outcomes of what might be called "real-life learning," as in measures of academic achievement. Typically these are about .5 and they can range upwards to as high as .8. It can be argued, of course, that academic achievement is largely the same thing as is measured with the tasks of intelligence tests, and therefore the prediction of the former from the latter is primarily only overlap in measurement. But this merely puts a somewhat different interpretation on the observations noted above. In sum, then, it seems that several kinds of learning are not highly predictive of various developmental outcomes which are identified as indicants of intelligence (and vice versa).

Jensen (e.g., 1970) has emphasized the abovementioned observation by specifying a theory of two intelligences, one identified with performances on short-term learning tasks, intelligence I, and one defined by commonly accepted (by professionals) measures of intelligence, intelligence II. This theory is sometimes discussed as if it were virtually equivalent to the theory of fluid intelligence (Gf) and crystallized intelligence $(\mathrm{Gc})$, as stated by Cattell $(1963,1971)$ and Horn $(1967,1968,1970,1972,1975)$. In particular there have been suggestions (e.g., Jensen, 1973) that intelligence I represents much the same thing as fluid intelligence. In fact, however, the Jensen theory and Gf-Gc theory have rather different empirical referents and implications and involve different explanatory concepts (particularly as pertains to origin in development). In Gf-Gc theory, short-term learning tasks are regarded as indicating a process peculiar to such learning, just as in Jensen's theory, but this process is believed to be largely independent of the $\mathrm{Gf}$ and $\mathrm{Gc}$ forms of intelligence. Indeed, a broad short-term acquisition factor (SAF) has now been found to be reliably independent of $\mathrm{Gf}$ and $\mathrm{Gc}$ functions in several studies (e.g., see the reviews and results of Cattell, 1971; Horn, 1970, 1975, 1976; Horn and Bramble, 1967; Rossman and Horn, 1972), although the learning tasks also have nonzero correlations with the Gf and Ge factors. Thus it seems that both Gf and Gc involve, or are predictive of, short-term learning, but much of the reliable variance of such learning tasks represents functions that are distinct from those usually accepted as highly indicative of intelligence.

Building on the findings of Botwinick and Storandt (1974), Horn (1975) has suggested that the SAF function is capacity, or effortfulness, of concentration in achieving primary memory. Perhaps this should be regarded as a form of intelligence (namely the intelligence I of Jensen's theory). If so it should be recognized as representing rather more elementary processes of thinking than are measured in Gf and $\mathrm{Gc}$ (even as is suggested in Jensen's theory). Shortterm learning is found early in evolutionary and child development and in this sense is by no means the sine qua non of human, adult intelligence. In any case, the essential point is that whereas the intelligences of $\mathrm{Gf}$ and $\mathrm{Gc}$ do indeed involve span of apprehension and short-term acquisition functions, there is reliable variance in SAF tasks which is neither of Gf or Gc and is itself cohesive in the sense that it forms a common factor.

A distinction between primary memory and secondary acquisition has been suggested by re- 
search on learning and cognitive processes in which individual differences were not the focus of concern (see Horn, 1974, 1975 for reviews). The distinction is indicated by the well-known primacy and recency effects in learning, for example, but it is suggested by several other findings as well (see Kintsch, 1970). Basically the notion is that primary memory (PM) indicates the individual's capacity to hold discrete items of information within the span of immediate apprehension without these items themselves being meaningfully associated. In the well-known dichotic listening research (Broadbent, 1954), for example, it is shown that in the very short term the person remembers by means of sensory descriptors (the ear at which the stimuli were sensed) rather than in terms of relationships among the stimuli (odd versus even numbers). Such memory, indicated by the recency effect, is evanescent, lasting perhaps no longer than 30 seconds if there is no rehearsal. If stimuli are apprehended in terms of meaningful descriptors, however, as indicated by the primacy effect, then the material can be retained for much longer periods of time. This is referred to here as secondary acquisition (SAC).

Collectively, the findings in this area of learning led Mandler (1967) to propose that the span memory which Miller (1965) colorfully described as a magical number $7 \pm 2$ for information processing is in fact comprised of a primary memory (PM) with span of roughly $4 \pm 1$ and a secondary process (SAC) with span of roughly 3 \pm 1 . These processes have been indicated clearly in research in which individual differences are treated as error, but there is no compelling evidence that there are notable and independent individual differences in the two processes. However, there is ample evidence of notable individual differences in span memory, as such (PM plus SAC). Also, we have at least one preliminary set of results showing that the primacy and secondary processes are reliably independent (Horn, Donaldson, Mason, Pisarowicz and Ward, 1975). Hence it seems likely that what has been shown to be of interest in extensive re- search on learning will be of relevance also for understanding individual differences in intellectual performances.

The suggestion is, therefore, that some forms of short-term learning depend very much on meaningful mediation (verbal mediation being perhaps the easiest to identify), whereas other forms of such learning involve this kind of mediation much less or not at all. The suggestion is, too, that these processes relate differently to Gf and Gc and represent a function (or functions) that is (are) independent of Gf and Gc. The present study was designed to follow up on these suggestions and attempt to make the relevant evidence a bit more definitive. At this point in the development of Gf-Gc theory it would seem that to the extent that an SAF task represents secondary acquisition and to the extent that this is mediated by the knowledge of advanced acculturation, to that extent the measure should relate more to Gc than to Gf or to a separate SAF factor. On the other hand to the extent that an SAF task represents secondary acquisition and mediation of the kind that involves perception of relationships that is not greatly enhanced by advanced acculturation, to that extent the SAF measure should relate more to Gf than to Gc. Thus SAC tasks can relate to either Gf or Gc and the principal determinant of which they relate to is the extent to which acculturation facilitates associations.

The hypotheses in respect to primary memory are more ticklish. It seems that primary memory must be involved in reasoning in which several aspects of a problem must be held in immediate awareness while one works out the relationships among the aspects in order to solve the problem. Such reasoning is very prominent in the variables which define Gf, but it must be present also in $\mathrm{Gc}$ in the acquisition of the concepts and aids of which this is comprised. To a lesser extent, this reasoning also must be present in some of the tasks which define it (verbal analogies, for example). Hence a case can be made for primary memory being prominently related to both $\mathrm{Gf}$ and Gc. It is not unequivocally clear that it 
should relate more to one than to the other, although it would seem that it might well have a stronger relationship to Gf because a larger proportion of the variance of this factor represents reasoning in the immediate situation (as opposed to in the past). In either case the evidence reviewed above (and in Horn, 1974, 1975, 1976) suggests that a substantial proportion of primary memory variance should be unrelated to either Gf or Gc.

\section{Methodological Rationale}

In broad overview, then, our purpose here is one of providing an improved description of the relationships among indicants of short-term apprehension and acquisition (retention being implied) and indicants of intelligence. But how best to try to describe these relationships given a collection of variables representing (ex hypothesi) the major kinds of influence? The thought was that perhaps Tucker's (1958) interbattery method of factor analysis might be particularly appropriate for this purpose. This procedure has been practically ignored in psychological research. Yet is seems to be analytically sound and to be based upon a rationale that provides a useful metatheory for some kinds of substantive issues. This rationale can be described roughly as follows.

One first supposes that sets of variables are usefully regarded as distinct. For example, the indicants of intelligence in psychometric tests can be regarded as distinct in a number of ways from the SAF variables derived from research on learning and cognitive processes. One then reasons that the variance of primary interest in these two sets of variables is that of the covariance between the two, exclusive of the covariance within each set. In the present research interest is focused on the processes which may be common to SAF variables and psychometric indicants of intelligence, excluding reliable variance within each set of variables considered separately. It is implied that the latter would contain systematic influences pertaining to testing devices or forms of measurement which, while interesting for some purposes, are best laid aside in a study focusing on the processes of SAF and intelligence. The methods do not preclude the possibility of identifying separate factors of SAF and intelligence; they merely force primary consideration on the covariance between the two classes of variables. Thus it would seem that with a proper selection of variables, application of Tucker's methods might indicate the distinction between primary memory and secondary acquisition, as well as the distinction between Gf and Gc. Since the methods also provide indications of the relationships between various factors, they can suggest the relationships of $\mathrm{Gf}$ and Gc to any SAF functions that might be indicated.

It can be argued also that because the interbattery methods have been used only very little in substantive research, it is worthwhile to study these methods, as such, by finding out how they work with variables that have a prominent place in psychological theory.

\section{Procedures}

\section{Subjects}

The data were gathered in the IXth form of a Higher Secondary School in Chandigarh, India. In all, 306 students were available for study, of which 265 were obtained in the final sample. Absences and failures in obtaining complete test results accounted for the loss of 41 potential subjects. No analyses were run to test hypotheses that the group of 41 omitted students was systematically different from the selected sample, since mere absences appeared to be unrelated to the testing. The median age of the students was about 14 years.

\section{Sampling of Variables}

A variety of miniature learning tasks was used in an effort to represent the SAF domain of variables. Because both paired associate and serial learning have been extensively studied, tasks representing both of these kinds of learning were sampled. Because verbal mediation was believed 
Table 1

Variables of SAF Learning and Intelligence

\begin{tabular}{|c|c|c|}
\hline Brief Description of Variable & $\begin{array}{l}\text { Variable } \\
\text { Symbol }\end{array}$ & $\begin{array}{l}\text { Primary } \\
\text { Factor } \\
\text { (if known) }\end{array}$ \\
\hline \multicolumn{3}{|l|}{ Learning Tasks } \\
\hline $\begin{array}{l}\text { 1. Paired Associate Learning: Nonsense syllables } \\
\text { for both stimulus and response }\end{array}$ & PANS - 2 & Ma \\
\hline $\begin{array}{l}\text { 2. Paired Associate Learning: Nonsense syllable } \\
\text { response for meaningful work }\end{array}$ & PANS-1 & $?$ \\
\hline $\begin{array}{l}\text { 3. Paired Associate Learning: Meaningful word } \\
\text { for both stimulus and response }\end{array}$ & PAM & Ma \\
\hline $\begin{array}{l}\text { 4. Paired Associate Learning: Meaningful figure } \\
\text { for both simulus and response }\end{array}$ & PAF & ? \\
\hline $\begin{array}{l}\text { 5. Paired Associate Learning: Numbers for both } \\
\text { stimulus and response }\end{array}$ & PAN & ? \\
\hline 6. Serial Learning of nonsense syllables & SLNS & $?$ \\
\hline 7. Serial Learning of meaningful words & SIM & $?$ \\
\hline 8. Serial Learning of meaningful figures & SLF & $?$ \\
\hline 9. Serial Learning of numbers & SLN & $?$ \\
\hline 10. Digit recall in order presented & DR & Mr \\
\hline \multicolumn{3}{|l|}{ Psychometric Tasks } \\
\hline 11. GMAT Synonyms Vocabulary & SV & $\mathrm{V}$ \\
\hline 12. GMAT Opposites for Words & $O P$ & $\mathrm{~V}$ \\
\hline 13. GMAT Classify Words & CLW & CMR \\
\hline 14. GMAT Inferences from Written Passages & INF & Rs \\
\hline 15. GMAT Following Directions & FD & $?$ \\
\hline 16. GMAT Verbal Analogies (mexied esoteric and common) & AN & CMR \\
\hline 17. GMAT Number Series & NS & I \\
\hline 18. IPAT Figure Series & FS & $\mathbf{I}$ \\
\hline 19. IPAT Classify Figures & $\mathrm{CF}$ & CFR \\
\hline 20. IPAT Matrices & MAT & CFR \\
\hline 21. IPAT Topology & TOP & $?$ \\
\hline
\end{tabular}

to be an important variable, each kind of learning was represented by tasks in which performance seemingly would be very much aided by such mediation and by tasks in which such aid would be less likely to be a noteworthy determinant of performance. Because figural, semantic and symbolic materials were used in obtaining psychometric indicants of intelligence, this variation on content was also used in specifying the learning variables. The learning variables selected on the basis of these considerations are described briefly in the upper section of Table 1.

The learning variables were pretesteci and revised in a pilot study to establish appropriate difficulty levels and adequate reliabilities. This work was based upon a sample of 150 form IX students drawn from a Higher Secondary School that was different from, but very similar to, the one used in the factor analyses.

The learning tasks were administered individually in the order SLM, PAM, SLNS, PANS-1, SLN, PANS-2, SLF, PAN, DR, PAF (see Table 1). Five minutes rest was given between each task and after five tasks had been completed a 20-minute break was given. Students were called from different classes in a predecided random manner, and they were encouraged not to discuss the testing with their classmates. It was believed that only very minimal variance was introduced as a result of students talking with each other about the testing.

The paired associates tasks were presented with an electric memory drum. Retention was 
checked using the anticipation method described by Duncanson (1964). The regulator of the memory drum was set to provide a presentation every two seconds, except in the PAF task where the subject was required to draw his response and thus rate was set manually in response to his response. A pause of six seconds separated each of the 15 trials.

The SLNS, SLM and SLN tasks were also presented using the memory drum. The SLF task was administered in a booklet in which one figure appeared on each page. Exposure time in each case was fixed at two seconds per stimulus. The test intervals for SLNS, SLM, SLN and SLF were $1.0,1.0,0.5$ and 1.25 minutes respectively.

For the reasons suggested by Jensen (1964) the learning tasks were in each case scored as the total number of correct responses given by the subject over the trials allowed (15 in the paired associate tasks, 12 in the serial learning tasks). Liberal standards were allowed in spelling or drawing of the response.

To obtain the digit recall measure, sets of from 3 to 12 digits were presented orally starting with the smallest set and moving in order to successively larger sets. The subject was instructed to reproduce the digits in precisely the same order as they were presented. In scoring, one point was given for every item recalled in the position it occupied in the presentation; the total score was the number of points earned.

To represent the psychometric indicants of intelligence, the seven subtests of Hundal's
GMAT, General Mental Ability Test (Singh, 1967), were used along with the four subtests of the IPAT Culture-Fair Intelligence Test, Scale 2 (Cattell and Cattell, 1960). These variables are described in the lower part of Table 1.

The tests were administered in groups of approximately 30 subjects per group in the classrooms the students met in for their academic work. In this testing, as well as the learning tasks, the students were assured that their results were to be used only for research purposes and would not be made available to anyone except themselves. It seemed that the students were well motivated to do only their own work and to do as well as they could.

\section{Results}

Learning curves and reliabilities were calculated. The results from these analyses are available from Hundal upon request. In general, they indicate adequate reliability for most variables and an upward trend in the learning tasks until the last trial. This suggests that learning had not yet reached asymptote.

For purposes of inter-battery analysis, the intercorrelations between the 11 psychometric indicants of intelligence and the 10 learning variables were obtained. These are shown in Table 2.

The inter-battery methods were applied for potential two-, three- and four-factor solutions even though the assumption was that a two-

Table 2

Correlations Between Intellfgence and Learning Variables $(N=265)$

\begin{tabular}{|c|c|c|c|c|c|c|c|c|c|c|c|}
\hline Variable & & PANS-2 & PANS -1 & $\overline{P A M}$ & $\overline{\mathrm{PAF}}$ & PAN & SLNS & SLM & SLF & $\overline{\text { SLN }}$ & $\overline{\mathrm{DR}}$ \\
\hline Synonyms & SV & 29 & 26 & 36 & 08 & 27 & 28 & 33 & 17 & 16 & 29 \\
\hline Opposites & $\mathrm{OP}$ & 23 & 24 & 31 & 07 & 26 & 28 & 27 & 12 & 13 & 16 \\
\hline Word Classification & CLW & 22 & 26 & 27 & 11 & 24 & 23 & 25 & 09 & 20 & 13 \\
\hline Inferences & INF & 24 & 26 & 30 & 02 & 21 & 23 & 24 & 08 & 22 & 20 \\
\hline Directions & FD & 32 & 30 & 32 & 13 & 27 & 27 & 32 & 23 & 28 & 19 \\
\hline Analogies & AN & 24 & 22 & 35 & 15 & 20 & 23 & 29 & 19 & 19 & 2 \\
\hline Number Series & NS & 23 & 18 & 28 & 05 & 21 & 21 & 22 & 18 & 21 & 12 \\
\hline Figure Series & FS & 19 & 21 & 17 & 11 & 24 & 18 & 26 & 24 & 23 & 22 \\
\hline Figure Classification & $\mathrm{CF}$ & 07 & 05 & 10 & 04 & 09 & -01 & 04 & 15 & 15 & 02 \\
\hline Matrices & MAT & 21 & 22 & 23 & 04 & 27 & 21 & 28 & 33 & 21 & 20 \\
\hline Topology & TOP & 19 & 16 & 15 & 08 & 18 & 19 & 22 & 22 & 14 & 10 \\
\hline
\end{tabular}

Note. With this size sample a correlation of .12 is significantly different from zero at the .05 level; one of .17 is significant at the .01 level. 
factor solution would suffice. In fact, the twofactor solution appeared to be best, both because it captured most of the common covariance and because it seemed to make the best sense from a theoretical viewpoint. Therefore, this is the solution employed here. The other solutions may be obtained from either of the authors upon request.

Using the procedures suggested by Tucker's presentation of the inter-battery method, the factor coefficient matrices for the learning and intelligence measures were rotated independently. Both the varimax and the promax (power set at five) criteria were tried. As would be expected, the results from the two kinds of rotation were very similar: the interpretations of the factors would be virtually the same for either. Because the varimax solution provides a somewhat more parsimonious representation of these results than does the oblique solution, it is shown in Tables 3 and 4 . The promax solution may be obtained from either of the authors upon request.

Factor scores based upon the solutions shown in Tables 3 and 4 were calculated using the direct procedure (Horn, 1965). The intercorrelations among these scores were then calculated to indicate the relationships between the dimensions representing components of learning and
Table 3

Factor Coefficients for Learning Variables

\begin{tabular}{clcc}
\hline & Variable & Factor & Coading \\
\hline 1. & PANS-1 & D \\
2. PANS-2 & .46 & .22 \\
3. PAM & .47 & .17 \\
4. PAF & .61 & .14 \\
5. PAN & .14 & .11 \\
6. SLNS & .40 & .30 \\
7. SLM & .49 & .13 \\
8. SLF & .49 & .28 \\
9. SLN & .11 & .59 \\
10. DR & .30 & .33 \\
Percent of common variance & .34 & .22 \\
\hline
\end{tabular}

those representing components of intellect. The relevant intercorrelations are provided in Table 5.

\section{Discussion}

It can be noted first that the results of Table 2 are consistent with much previous research showing correlations of about .30 or lower between short-term learning variables and indicants of intelligence. The reliabilities of the learning and psychometric variables are in excess of .7 , and so in theory could have substantially larger correlations than are found here. Thus, these results again verify the general finding of considerable independence between learning and the various indicants of intelligence.

Table 4

Varimax Factor Coefficients for Intelligence Variables

\begin{tabular}{lllcc}
\hline & Variable & & \multicolumn{2}{c}{ Factor Loading } \\
\hline 11. GMAT Synonyms Vocabulary & SV & .52 & .20 \\
12. GMAT Opposites & OP & .49 & .09 \\
13. GMAT Word Classification & CLN & .45 & .11 \\
14. GMAT Inferences & INF & .48 & .09 \\
15. GMAT Directions & FD & .47 & .32 \\
16. GMAT Analogies & AN & .45 & .24 \\
17. GMAT Number Series & NS & .35 & .23 \\
18. IPAT Figure Series & FS & .25 & .40 \\
19. IPAT Figure Classification & CF & -.01 & .25 \\
20. IPAT Matrices & MAT & .25 & .48 \\
21. IPAT Topology & TOP & .21 & .31 \\
Percent of Common Variance & & 66.70 & 33.30 \\
\hline
\end{tabular}


Table 5

Intercorrelations Between Inter-Battery Factors

\begin{tabular}{lccc}
\hline & \multicolumn{2}{c}{$\begin{array}{c}\text { Factor C } \\
\text { (SAC) }\end{array}$} & $\begin{array}{c}\text { Factor D } \\
\text { (PM) }\end{array}$ \\
Factors & (SA & .40 \\
Factor A (GC) & .69 & .49 \\
Factor B (Gf) & .44 & .49 \\
\hline
\end{tabular}

Secondly, the results of Table 4 indicate again the commonly-found distinction between fluid and crystallized intelligence. Gc, the broader of the two factors, is defined primarily by Verbal Comprehension and Semantic Reasoning (CLN, INF and AN), but it also has a noteworthy relationship to Number Series, which often is a marker for the Induction primary ability. It would seem that the crystallized skills of mathematics are involved to a considerable extent in Number Series, and that this accounts for the finding that this variable has a relatively larger loading on Gc and smaller loading on Gf than is indicated for Figure Series, which also is an indicant of the Induction primary ability. As in several previous studies (e.g., Cattell, 1971; Horn, 1970, 1972, 1976), the Gf factor is defined primarily by Matrices and other Figural Reasoning tasks, but also has noteworthy correlations with Analogies and Number Series. Here, too, the factor is found to be significantly related to the Following Directions task.

Thus, findings for the psychometric devices are mainly of interest in showing replication of previous work. Of more interest for the evolution of knowledge in this area are the results for the learning variables. The interpretation which should be given to these findings is by no means obvious.

Of possible primary importance is the fact that the proportion of common variance for factor $\mathrm{C}$ is over twice that for factor $\mathrm{D}$. Since on $a$ priori grounds the principal variance in common between the variables of learning and intellect-the variance analysed-is believed to represent meaningful associations and learning mediated by such comprehension, it can be argued that factor $\mathrm{C}$ does indeed represent mainly an influence of mediation in the learning variables. This would link the factor at least roughly to the secondary acquisition process (SAC) discussed in the theoretical introduction of this paper. Such an interpretation is supported by the fact that in both paired associate and serial learning, the tasks most likely to involve associations have their major variance in factor $C$ rather than in factor $D$. This observation must be qualified by the fact that in each case the meaningfulness of the tasks is that of the culture and thus the variance also reflects the acculturation of Gc. This is perhaps emphasized in the convergence brought about by the inter-battery procedures (although recall that we are looking at the rotated results, not the principal components). The observation needs to be qualified also by the fact that both of the paired associate tasks based upon nonsense materials (PANS-1 and PANS-2) have notably higher correlations with factor $\mathrm{C}$ than with factor D. This finding may be consistent with Eysenck's (1967) contention that there is a " . . dependence of paired associate learning on verbal mediation, in contrast to the rote character of serial learning ..." It is not unreasonable to suppose that a considerable amount of variance on tasks involving even nonsense syllables is produced by individual differences in recognizing the stimuli as possible parts of words or as having associations which are aided by acculturation. In any case, it seems that in factor $\mathrm{C}$ there is emphasis on learning mediated by meaningful associations, even as the process may be somewhat more complicated than this. Tentatively, therefore, it is interpreted as representing mainly secondary acquisition (SAC).

Following the lead suggested above and in previous sections of this report, factor $D$ might be interpreted as representing primary memory (PM). This is supported by the fact that the factor has small variance relative to factor $\mathrm{C}$ and that this is particularly true for variables in which meaningful associations are most possible. The DR variable, representing the span memory of $7+2$ comprised of primary memory and secondary acquisition, splits its variance between factor $C$ and factor $D$, as expected if indi- 
vidual differences in the two processes are distributed independently. Similarly, the variance of other learning tasks is split in a way to suggest a dominant influence of secondary acquisition and a less pronounced influence of primary memory.

A case might be made for supposing that factor $\mathrm{D}$ represents memory with figural materials, which also are emphasized in factor B, interpreted as Gf. Support for such an interpretation certainly is provided by the prominent loading of SLF on factor D. But contrary to this hypothesis is the fact that the other learning variables of the factor are not figural and PAF correlates only .11 with the factor (although it must be granted that the communality for this latter variable is very small and thus the variable may represent mainly a random influence relative to the other variables of this study).

For present purposes factor D is tentatively interpreted as mainly representing primary memory (PM), although that this interpretation is debatable and must be supported by further research before it can be accepted as compelling.

Given these tentative interpretations, the results of Table 5 can be viewed as an indication of the extent to which SAC and PM are implicated in Gf and Gc respectively. As expected, SAC is more highly related to Gc than to Gf. Although the correlational results of Table 5 are partly only a restatement of the results produced by the inter-battery procedures, it is of some interest to note that when the $r$ coefficients are treated as those for different variables the difference between the Gc-SAC correlation of .69 and GfSAC correlation of .44 is found to be significant. The difference between the Gc-PM and Gf-PM correlations, however, is not significant.

The findings thus indicate that mediated learning is an aspect of both $\mathrm{Gf}$ and $\mathrm{Gc}$, but may be implicated to a greater extent in Gc than in Gf. It is possible, however, that this latter finding mainly reflects the fact (ex hypothesi) that it is easier for experimenters to create tasks in which mediation is aided by the acculturation of
Gc than it is to create tasks in which this is not true. Primary memory, also, is an aspect of both the Gf and Gc forms of intelligence. In this case the involvements of PM in Gf and $\mathrm{Gc}$ are about the same.

\section{Summary}

The major implications of this research can be briefly summarized as follows:

1) Psychometric indicants of intelligence have notable, but not substantial, variance in common with the short-term learning variables studied most intensively in some 50 years of research on learning.

2) The major common variance between psychometric indicants of intelligence and shortterm learning variables represents meaningful associations and learning mediated by such associations.

3) To a lesser extent intelligence (as indicated by psychometric indicants) involves the span of apprehension of primary memory.

4) Two forms of intelligence, fluid intelligence (Gf) and crystallized intelligence ( $\mathrm{Gc}$ ), involve primary memory to about the same extent, but acquisition mediated by meaningful associations is involved more in $\mathrm{Gc}$ than in Gf.

5) Span memory, measured by the number of digits recalled immediately after presentation, is made up of the two components mentioned above, primary memory and secondary acquisition, and on this basis also has variance partly in Gf and partly in Gc.

\section{References}

Allison, R. B. Learning parameters and human abilities. Office of Naval Research Technical Report. Princeton, N. J.: Educational Testing Service, 1960.

Birren, J. E. and Woodruff, D. S. Human development over the life span. Ch. 13 in Life-span Developmental Psychology: Personality and Socialization. P. B. Baltes and K. W. Schaie (Eds.). New York: Academic Press, 1973, 305-337.

Botwinick, J. and Storandt, M. Memory, Related Functions and Age. Springfield, Ill.: Thomas, 1974. 
Broadbent, D. C. The role of auditory localization in attention and memory span. Journal of Experimental Psychology, 1954, 45, 191-196.

Carroll, J. B. Fitting a model of school learning to aptitude and achievement data over grade levels. In The Aptitude-Achievement Distinction. D. R. Green (Ed.). New York: McGraw-Hill, 1975.

Carver, R. P. and DuBois, P. H. The relationship between learning and intelligence. Journal of Educational Measurement, 1967, 4, 133-136.

Cattell, R. B. Theory of fluid and crystallized intelligence: a critical experiment. Journal of Educational Psychology, 1963, 54, 1-22.

Cattell, R. B. Abilities: their Structure, Growth and Action. Boston: Houghton-Mifflin, 1971.

Cattell R. B. and Cattell, A. K. S. Handbook for the Culture Fair Intelligence Test, Scale 2. Institute Personality and Ability Testing. Illinois, 1960.

Duncanson, J. P. Intelligence and the ability to learn. Office of Naval Research Technical Report. Princeton, N. J.: Educational Testing Service, 1964.

Eysenck, H. J. Intelligence assessment: a theoretical and experimental approach. British Journal of Educational Psychology, 1967, 37, 81-98.

Fleishman, E. A. and Rich, S. The role of kinesthetic and visual-spatial abilities in perceptual-motor learning. Journal of Experimental Psychology, $1963,66,6-11$.

Guilford, J. P. The Nature of Human Intelligence. New York: McGraw-Hill, 1967.

Harootunion, B. Intelligence and the ability to learn. Journal of Educational Research, 1966, 59, 211. 214.

Horn, J. L. A rationale and test for the number of factors in factor analysis. Psychometrika, 1965, 30, 179-185.

Horn, J. L. Intelligence-Why it grows, why it declines. Trans-Action, 1967, 5, 23-31.

Horn, J. L. Organization of abilities and the development of intelligence. Psychological Review, 1968, 75, 242-259.

Horn, J. L. Organization of data on life-span development of human abilities. Ch. 16 in Life-Span Developmental Psychology, L. R. Goulet and P. B. Baltes (Eds.). New York: Academic Press, 1970, 423-466.

Horn, J. L. The structure of intellect: primary abilities. Ch. 12 in Multivariate Personality Research. R. H. Dreger (Ed.), Baton Rouge, La.: Claitor, 1972, 541-611.

Horn, J. L. A study of speed, power, carefulness, and short-term learning components of intelligence and changes in these components in adulthood development. Research Proposal submitted to Na- tional Science Foundation. GB-41452, 1974.

Horn, J. L. Psychometric Studies of Aging and Intelligence. In S. Gershon and A. Raskin (Eds.) Genesis and Treatment of Psychological Disorders in the Elderly. New York: Raven Press, 1975, 1943.

Horn, J. L. Human abilities: a review of research and theory in the early 1970s. Annual Review of Psychology, 1976 (in press).

Horn, J. L. and Bramble, W. J. Second order ability structure revealed in rights and wrongs scores. Journal of Educational Psychology, 1967, 58, 115122.

Horn, J. L., Donaldson, G., Mason, R., Pisarowicz, J. and Ward, C. An interim report on a study of short-term learning, speediness and intellectual variables. University of Denver, 1975.

Jensen, A. R. Individual differences in learning: Interference factor. Cooperative Research project No. 1867, U. S. Office of Education, 1964.

Jensen, A. R. A theory of primary and secondary familial mental retardation. In N. R. Ellis (Ed.) International Review of Mental Retardation, Vol. 4. New York: Academic Press, 1970, 33-105.

Jensen, A. R. Educability and Group Differences. New York: Harper and Row, 1973.

Kintsch, W. Learning, Memory and Conceptual Processes. New York: Wiley, 1970.

Mandler, G. Organization and memory. In K. W. Spence and J. T. Spence (Eds.) The Psychology of Learning and Motivation: Advances in Research and Theory, Vol. 1. New York: Academic Press, 1967, 328-372.

Miller, G. A. The magical number seven, plus or minus two: some limits on our capacity for processing information. Psychological Review, 1956, 63, 81-97.

Rossman, B. B. and Horn, J. L. Cognitive, Motivational and Temperamental Indicants of Creativity and Intelligence, Journal of Educational Measurement, 1972, 9, 265-286.

Singh, A. A study of reliability and validity of Hindi version of "Hundal General Mental Ability Test." Psychological Studies, 1967, 12, 83-90.

Stevenson, H. W., Friedrichs, A. G. and Simpson, W. E. Interrelations and correlates over time in children's learning. Child Development, 1970, 41, 625-637.

Tucker, L. R. An interbattery method of factor analysis. Psychometrika, 1958, 23, 111-136.

Tucker L. R. Application of three-mode factor analysis to learning variables, 1963.

Woodrow, $\mathrm{H}$. The relation between abilities and improvement with practice. Journal of Educational Psychology, 1938, 29, 215-230. 


\section{Acknowledgements}

This research was supported in part by grants from the National Science Foundation, Gb-41452 and the Army Research Institute, DACH19-74-G-0012. The authors acknowledge Mark Foster's insightful help in applying computer programs for these analyses, and in interpreting results.

\section{Authors' Addresses}

P.S. Hundal, Guru Nonak University, Amritsar, India.

John L. Horn, Department of Psychology,

University of Denver, Denver, CO 80210.

\section{Manuscripts Accepted for Publication}

JERARD KEHOE and THOMAS J. REYNOLDS (Department of Psychology, University of University of Southern California, University Park, Los Angeles, CA 90007). Interactive Multidimensional Scaling of Cognitive Structure Underlying Person Perception.

OLIVER TZENG (Department of Psychology, Indiana University-Purdue University at Indianapolis, 1201 E. 38th Street, Indianapolis, IN 46205). A Quantitative Method for Separation of Semantic Spaces.

EDWARD HELMES (Department of Psychology, The University of Western Ontario, London, Ontario, Canada N6A 5C2) and DOUGLAS N. JACKSON. The Item Factor Structure of the Personality Research Form.

DOMENIC V. CICCHETTI (Veterans Administration Hospital, West Spring Street, West Haven, CT 06516) and JOSEPH L. FLEISS. Comparison of the Null Distribution of Weighted Kappa and the C-Ordinal Statistic.

JUM C. NUNNALLY (Department of Psychology, Vanderbilt University, 134 Wesley Hall, Nashville, TN 37203), CHARLES L. LEMOND, and WILLIAM H. WILSON. Studies of Voluntary Visual Attention-Theory, Research Methods and Psychometric Issues.

JAMES MACKEY and ANDREW AHLGREN (Center for Educational Development, Office of the Vice President, Academic Affairs, 317 Walter Library, University of Minnesota, Minneapolis, MN 55455). Dimensions of Adolescent Alienation.

FUMIKO SAMEJIMA (Department of Psychology, Ayres Hall, University of Tennessee, Knoxville, TN 37916). A Use of the Information Function in Tailored Testing.

PANG-CHIEH LIN and LLOYD G. HUMPHREYS (425 Psychology Building, University of Illinois, Champaign, IL 61820). Predictions of Academic Performance in Graduate and Professional School.

BENJAMIN D. WRIGHT (Department of Education, University of Chicago, 5835 Kimbark Avenue, Chicago, IL 60037) and GRAHAM DOUGLAS. Best Procedures for Sample-Free Item Analysis.

NANCY E. BETZ (Department of Psychology, The Ohio State University, 1945 N. High Street, Columbus, $\mathrm{OH} 47210)$. Effects of Immediate Knowledge of Results and Adaptive Testing on Ability Test Performance. 